\title{
Time trends of chest pain symptoms and health related quality of life in coronary artery disease Anna Kiessling* and Peter Henriksson
}

\author{
Address: Karolinska Institutet, Department of Clinical Sciences, Danderyd Hospital, Stockholm, Sweden \\ Email: Anna Kiessling* - anna.kiessling@ki.se; Peter Henriksson - peter.henriksson@ki.se \\ * Corresponding author
}

Published: 6 March 2007

Health and Quality of Life Outcomes 2007, 5:13 doi:10.1 186/1477-7525-5-13

This article is available from: http://www.hqlo.com/content/5/1//3

C) 2007 Kiessling and Henriksson; licensee BioMed Central Ltd.

This is an Open Access article distributed under the terms of the Creative Commons Attribution License (http://creativecommons.org/licenses/by/2.0), which permits unrestricted use, distribution, and reproduction in any medium, provided the original work is properly cited.
Received: 6 December 2006

Accepted: 6 March 2007

\begin{abstract}
Background: There is at present a lack of knowledge of time trends in health related quality of life (HRQL) in common patients with coronary artery disease (CAD) treated in ordinary care. The objective of this study is to assess and compare time trends of health related quality of life (HRQL) and chest pain in patients with coronary artery disease.

Methods: 253 consecutive CAD patients in Stockholm County, Sweden - 197 males/56 females; $60 \pm 8$ years - were followed during two years. Perceived chest pain symptoms and three global assessments of HRQL were assessed at baseline, after one and after two years. EuroQol-5 dimension (EQ-5D) with a predefined focus on function and symptoms; the broader tapping global estimates of HRQL; EuroQol VAS (EQ-VAS) and Cardiac Health Profile (CHP) were used. Chest pain was ranked according to Canadian Cardiovascular Society (CCS). Change in HRQL was analysed by a repeated measurements ANOVA and chest pain symptoms were analysed by Friedman non-parametric ANOVA.

Results: Perceived chest pain decreased during the two years ( $p<0.00022)$; CCS 0: 4I-5I\%; CCS I: 19-15\%; CCS 2: 3I-27\%; CCS 3: 5-4\% and CCS 4: 4-2\%. By contrast, HRQL did not change: EQ-5D: 0.76 (Cl 0.73-0.79) -0.78 (Cl 0.75-0.8I), EQ-VAS: 0.68 (Cl 0.66-0.7I)-0.68 (Cl 0.65-0.7I) and CHP: $0.66(\mathrm{Cl} 0.64-0.69)-0.66(\mathrm{Cl} 0.64-0.69)$.

Conclusion: $\mathrm{HRQL}$ did not increase despite a reduction in the severity of chest pain during two years. This implies that the major part of HRQL in these consecutive ordinary patients with CAD is unresponsive to change in chest pain symptoms.
\end{abstract}

\section{Background}

Disease specific and all-cause mortality in patients with coronary artery disease (CAD) have declined during the last decades [1]. Patients with CAD have at present well treated physical symptoms and cardiovascular drugs help to reduce their risk of a new coronary event. However, the patient's perception of disease is to a large extent unexplored. Health related quality of life (HRQL) is often added as an outcome variable in randomised intervention clinical trials with the aim to assess the patient's perception of the disease. HRQL is - in contrast to morbidity and mortality - a multidimensional construct without a golden standard or definition. HRQL is always assessed and defined by the individual patient. Objective - e g patency - and subjective - e g HRQL - results are often in conflict $[2,3]$. Some studies report improved perceived 
function and wellbeing after an intervention resulting in similar levels of HRQL as in normative populations $[4,5]$. By contrast, in other studies patients examined e. g. four years after an acute myocardial infarction still had a decreased HRQL as compared to community norms [6]. A problem with randomised controlled trials is that they often include highly selected patient groups. This selection decreases the ability to extrapolate the effects, to the unselected cohorts in routine care. Descriptive studies of unselected populations with CAD receiving routine clinical care are scarce. Studies examining time trends in such populations are consequently even more scant. However, in order to understand the patient perspective - illness of CAD it is mandatory to study HRQL in unselected cohorts of patients. The aim of our study is to assess time trends in chest pain symptoms and HRQL in routine care patients with CAD.

\section{Methods \\ Design and subjects}

We identified a cohort of consecutive patients with CAD and assessed chest pain symptoms and perceived quality of life during two years. This was done in connection with a randomised study showing that participatory learning of secondary prevention for general practitioners decreased cholesterol levels in their patients with coronary artery disease [7].

The Department of Medicine at Södertälje Hospital, located in the southernmost part of Stockholm County, Sweden, provides planned and emergency health care for acute and elective cardiac patients in a catchments area of approximately 95000 habitants. All in- and outpatients with an age less or equal to 70 years who had visited the department during the preceding year, due to symptoms and signs of CAD (ICD-9 code 410-414) were identified (1995). The age limit was chosen due to lack of secondary preventive studies in older patients at time of recruitment. 429 patient records were investigated. 106 patients were excluded, the majority because they did not fulfil the criteria of CAD and a few because of other life threatening disease or because they lived outside the catchments area. 68 of the remaining 323 patients fulfilling the criteria for inclusion refused to participate.

Criteria for a confirmed diagnosis of CAD in the patient record were:

I: A diagnosis of angina pectoris, either by objective criteria based on coronary angiography, or a pathologic exercise- or stress-test, or a clinical assessment based on typical angina symptoms at exercise with or without ECG-evidence of possible or definite ischemia.
II: A diagnosis of myocardial infarction based on either WHO-criteria [8] or on unequivocal ECG-findings.

Hypertension was considered to be present if systolic blood pressure was $>140 \mathrm{~mm} \mathrm{Hg}$, if diastolic blood pressure was $>90 \mathrm{~mm} \mathrm{Hg}$ or if antihypertensive medication was used. Diabetes mellitus was defined as fasting plasma glucose of $\geq 7.0 \mathrm{mmol} / \mathrm{l}$, a self-reported history of diabetes mellitus or treatment for diabetes.

The patients were examined and they answered questionnaires including symptoms of angina pectoris and HRQL assessments at inclusion, after one respectively after two years. Baseline characteristics are given in Table 1 and the trial profile in figure 1. Further details of the patient cohort, the inclusion procedure and two of the health related quality of life instruments used in this study; the Cardiac Health Profile questionnaire (CHP) and the EuroQol-VAS instrument (EQ-VAS) are available in a previous publication [9]. There were no significant differences in assessed total CHP scores between patients with or without a prior myocardial infarction or between those who had or had not passed an intervention - Percutaneous Coronary Intervention (PCI)/Coronary Artery Bypass Surgery (CABG) - at baseline. Furthermore, a regression analysis showed no influence on HRQL of duration of CAD or age [9].

The HRQL-questionnaires were self-completed. The Swedish versions of the CHP- and EQ-instruments were used. One research nurse performed all interviews and physical examinations at the department. She also handled all case report forms. All participating patients gave written informed consent. The study complies with the Declaration of Helsinki and was approved by the ethics committee of Karolinska Institutet at Huddinge University Hospital.

\section{Assessment of chest pain symptoms and health related quality of life}

The patients ranked existence and degree of current angina symptoms according to the Canadian Cardiovascular Society classification (CCS) $0-4[10]$ at baseline, after one respectively two years (Table 2 ).

The definition of HRQL in this study is that 'quality of life' in clinical medicine represents the functional effect of an illness and its consequent therapy upon a patient, as perceived by the patient [11].

The Cardiac health profile questionnaire (CHP) is a disease-specific HRQL questionnaire specially designed for respondents with CAD. CHP assesses the HRQL in a broad perspective. The questionnaire includes questions about physical function/general health, emotional, social 
Table I: Patient characteristics at baseline

\begin{tabular}{|c|c|}
\hline Patients included (n) & 253 \\
\hline Age (years) & $60.1 \pm 7.5$ \\
\hline $\mathrm{BMI}\left(\mathrm{kg} / \mathrm{m}^{2}\right)$ & $28 \pm 4.2$ \\
\hline Waist/hip ratio & $0.95 \pm 0.07$ \\
\hline Systolic blood pressure (mmHg) & $139 \pm 20$ \\
\hline Diastolic blood pressure $(\mathrm{mmHg})$ & $84 \pm 9$ \\
\hline Duration of CAD (years) & $6.0 \pm 5.6$ \\
\hline Total lipoprotein cholesterol & $6.4 \pm 1.1$ \\
\hline Triglycerides & $2.1 \pm 1.1$ \\
\hline High density lipoprotein cholesterol & $1.2 \pm 0.3$ \\
\hline Low density lipoprotein cholesterol & $4.2 \pm 1.0$ \\
\hline Men/Women & $197(78 \%) / 56(22 \%)$ \\
\hline Family history of CAD & $97(38 \%)$ \\
\hline Diabetes mellitus & $37(15 \%)$ \\
\hline Hypertension & $67(26 \%)$ \\
\hline \multicolumn{2}{|l|}{ Smoking } \\
\hline Non smoker & 107 (42\%) \\
\hline Ex-smoker & $85(34 \%)$ \\
\hline Current smoker & $61(24 \%)$ \\
\hline History of myocardial infarction & $167(66 \%)$ \\
\hline History of coronary artery bypass grafting & $95(38 \%)$ \\
\hline History of $\mathrm{PCl}$ & $29(11 \%)$ \\
\hline History of peripheral arterial disease & $5(2 \%)$ \\
\hline History of cerebrovascular lesions & $3(1 \%)$ \\
\hline Use of Acetyl salicylic acid & $205(81 \%)$ \\
\hline Use of $\beta$-blockers & $165(65 \%)$ \\
\hline Use of Lipid lowering drugs & $49(19 \%)$ \\
\hline Other co-morbidity* & $71(28 \%)$ \\
\hline
\end{tabular}

Values are given as mean \pm SD or n (\%); BMI, Body Mass Index; CAD, Coronary Artery Disease; PCl, Percutaneus Coronary Intervention; *For example locomotive, gastro intestinal, pulmonary, depression, renal calculi and prostate disorders

and cognitive function. The 16 items in the CHP questionnaire (English version) are shown in Table 3. For further details of content, development, validity, reliability and sensitivity in Swedish cohorts of patients with CAD see previous publications $[9,12,13]$. The CHP yields an estimate of HRQL between 0 and 100 were 0 is equal to full health and 100 is equal to worst imaginable state. We have in this study transformed the individual global CHP mean scores to a $0-1$ scale were 0 is equal to death and 1 is equal to full health. This was done in order to facilitate comparison to the EuroQol results.

Multivariate explorative factor analytical methods were used to identify the underlying structure as described in a previous publication [9]. Four independent principal components - sub-domains - of HRQL were identified. These components are physical function/general health, emotional, social and cognitive function.

The generic HRQL questionnaires EuroQol-5 Dimensions (EQ-5D) self-classifier and EuroQol-VAS (EQ-VAS) were used to test the robustness of the analysis of time trends of HRQL as measured by the CHP questionnaire. In the EQ$5 \mathrm{D}$ self classifier the respondents classified their own health status in five aspects - mobility, self-care, usual activities, pain/discomfort and anxiety/depression into one of three levels - no problems, moderate problems and severe problems $[14,15]$. Each obtained individual health state was then given an index value (0-1) based on a method described by Dolan [16]. Since there is no Swedish tariff for EQ5D-index, the UK EQ5D-index tariff was used to calculate the EQ5D-index values, were 0 is equal to death and 1 is equal to full health [16]. Health states worse than death were given a score of 0 since the scaling of negative health states are controversial $[17,18]$.

In the EQ-VAS method the respondent marks present HRQL on a $20 \mathrm{~cm}$ vertical measurement scale with labelled anchors 'dead' (0) and 'full health' (100). The individual numbers marked on the scale were divided by 100 in order to obtain EQ-VAS scores between 0 and 1. Both EQ5D methods are easy and rapid generic instruments, well validated and found to be reliable in different cultures and in different diseases including a new validation study in patients after myocardial infarction $[19,20]$.

\section{Statistics}

The main outcome variable in the present study is change in HRQL and chest pain (CCS) over time. HRQL was analysed by a repeated measurements ANOVA and chest pain 


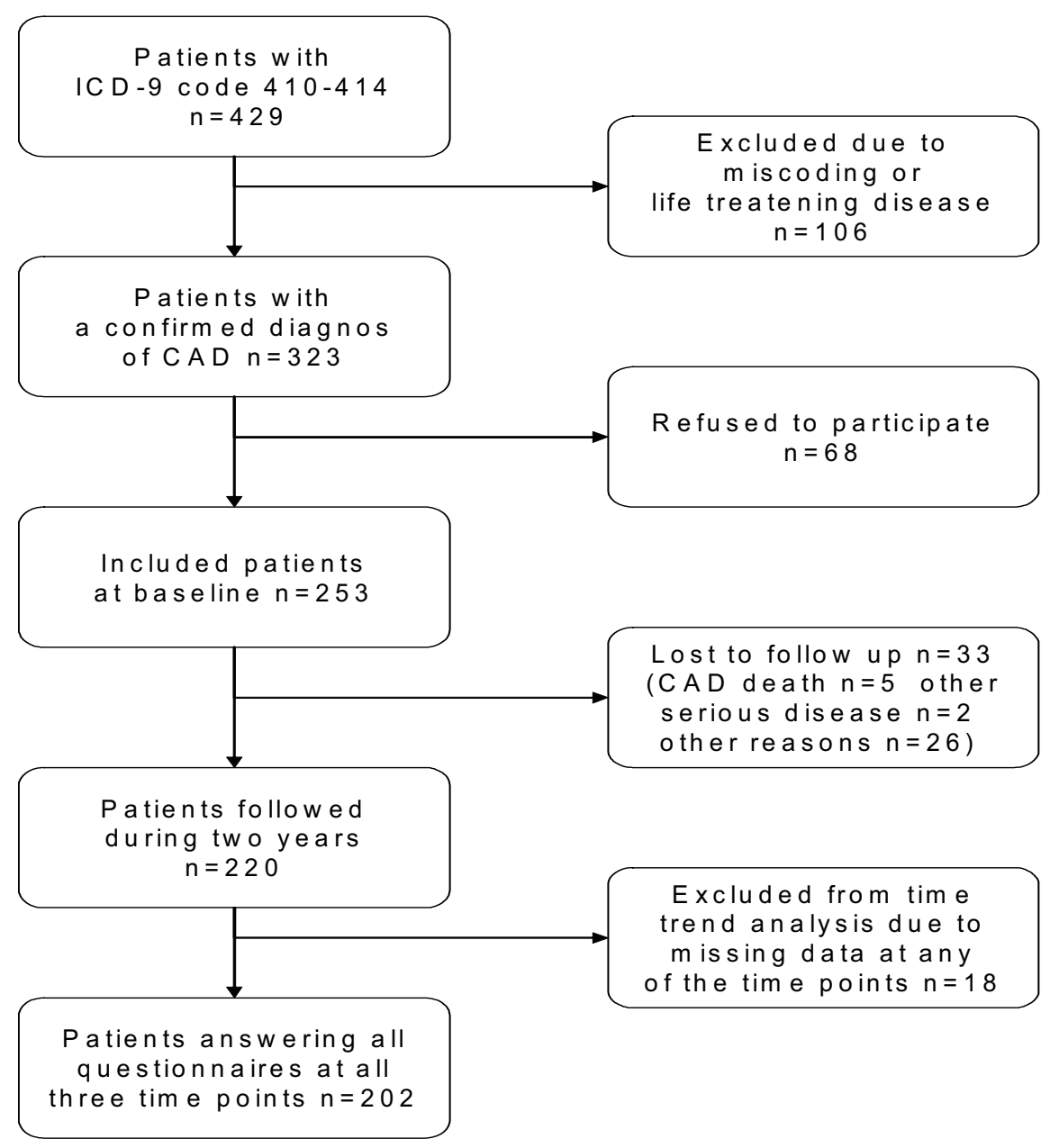

Figure I

Trial profile. Inclusion, participation, dropouts and follow up.

symptoms were analysed by Friedman non-parametric ANOVA. The underlying structure of HRQL as assessed by $\mathrm{CHP}$ was explored by the factor analytic multivariate technique, principal component (PC) analysis. The 16 item variables were reduced into principal components, i.e. to avoid an influence of multicollinearities and to find orthogonal (independent) factors explaining the variance in the sample $[21,22]$. Another way to put this is to find independent underlying factors explaining the variation in answers. Varimax rotation was used. The Kaiser criterion was used to demarcate the total number of factors (principal components) to retain. Only factors with Eigen values greater than 1 were retained; i.e. unless a factor extracts at least as much as the equivalent of one original variable, it was dropped. We have described the details in a previous publication [9]. The factor coefficients of the four extracted PC at baseline were used to calculate PC:s at one respectively two years. The coefficients and the for- mula used can be obtained from the corresponding author. We used Spearman Rank order correlation analysis to assess whether a co variation between chest pain symptoms and HRQL existed. The descriptive data for the HRQL measurements are given as mean and 95\% confidence interval. The patient's baseline characteristics are given as mean \pm SD or $n(\%)$. The power of the present study is $80 \%$ to detect a change in EQ5D by $5 \%$.

\section{Results}

The characteristics of the study population at baseline are described in Table 1. 253 consecutive patients (197 males and 56 females) with a mean age of $60.1 \pm 7.5$ years were included. The response rates were $99 \%$ in all of the used HRQL instruments at baseline. The response rate was $98 \%$ on CCS. During the study five patients died and all of those due to cardiovascular disease. In addition, two patients had to be excluded due to another serious disease 
Table 2: Prevalence and severity of chest pain symptoms ranked according to the Canadian Cardiovascular Society classification (CCS 0-4)

\begin{tabular}{|c|c|c|c|c|c|}
\hline $\mathrm{n}=202$ & & Baseline & After I year & After 2 years & $\mathrm{P}<0.00022 *$ \\
\hline $\operatorname{ccs} 0$ & No chest pain & $82(41 \%)$ & $105(52 \%)$ & $104(51 \%)$ & \\
\hline CCS I & $\begin{array}{l}\text { Ordinary physical activity does not cause angina. Strenuous, rapid, or prolonged } \\
\text { exertion causes angina }\end{array}$ & $39(19 \%)$ & $28(14 \%)$ & $30(15 \%)$ & \\
\hline $\operatorname{ccs} 2$ & Slight limitation of ordinary activity (special circumstances) due to angina & $62(31 \%)$ & $54(27 \%)$ & $55(27 \%)$ & \\
\hline $\operatorname{cCS} 3$ & Marked limitation in ordinary physical activity due to angina & II (5\%) & $10(5 \%)$ & $9(4 \%)$ & \\
\hline $\operatorname{ccs} 4$ & $\begin{array}{l}\text { Unable to perform any physical activity without discomfort - angina may be } \\
\text { present at rest. }\end{array}$ & $8(4 \%)$ & $5(2 \%)$ & $4(2 \%)$ & \\
\hline
\end{tabular}

Friedman non-parametric ANOVA

and eight patients had moved out of the district and 18 patients refused participation (figure 1). Thus, we had altogether 220 patients at the two years follow-up (88\%). 202 patients answered CHP, EQ 5D, EQ-VAS and CCS at all three assessments points ( $81 \%$ of all included patients at baseline). There were no significant differences in baseline characteristics between responders and non-responders.

\section{Time trends}

The prevalence and severity of chest pain symptoms according to CCS grade - decreased during the two study years as shown in Table $2(\mathrm{p}<0.00022)$. By contrast, no significant change occurred in HRQL during the two years, as assess by three different instruments (figure 2). Furthermore no change at all was noted in any of the four major components of HRQL - cognitive, physical/general health perception, social and emotional function - derived from the CHP-instrument; (data not shown). During the study
$67(33 \%)$ of the patients contracted non cardiovascular co-morbidity. Analysis with inclusion or exclusion of these 67 patients did not change our results.

An analysis of co-variation between chest pain symptoms and HRQL showed significant correlations between CCS and all three global assessments of HRQL at all three time points, Table $4(\mathrm{p}=0.000000)$.

A similar analysis of the four independent sub-domains principal components - showed a correlation between chest pain symptoms and the physical/general health domain at all three time points $(\mathrm{p}=0.000000)$. By contrast, there was no correlation between cognitive function respectively social function domains and chest pain symptoms at any of the time points (Table 4). Figure 3 and 4 illustrates the different patterns of co-variation between chest pain and the cognitive respectively physical/general health domains of HRQL at baseline and at two years.

Table 3: Items in the Cardiac Health Profile questionnaire (CHP)

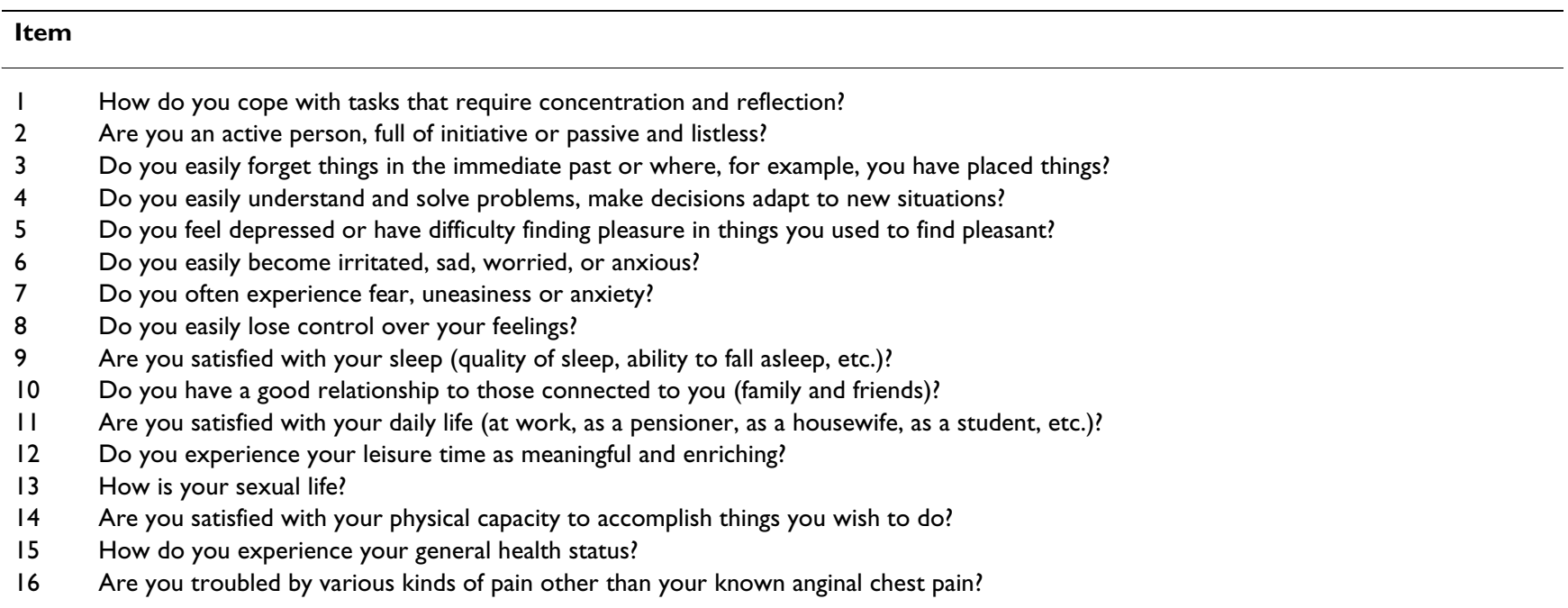

The answers are marked on a $100 \mathrm{~mm}$ VAS-scale with verbal anchors expressing extremes. High scores indicates a bad Health Related Quality of Life 


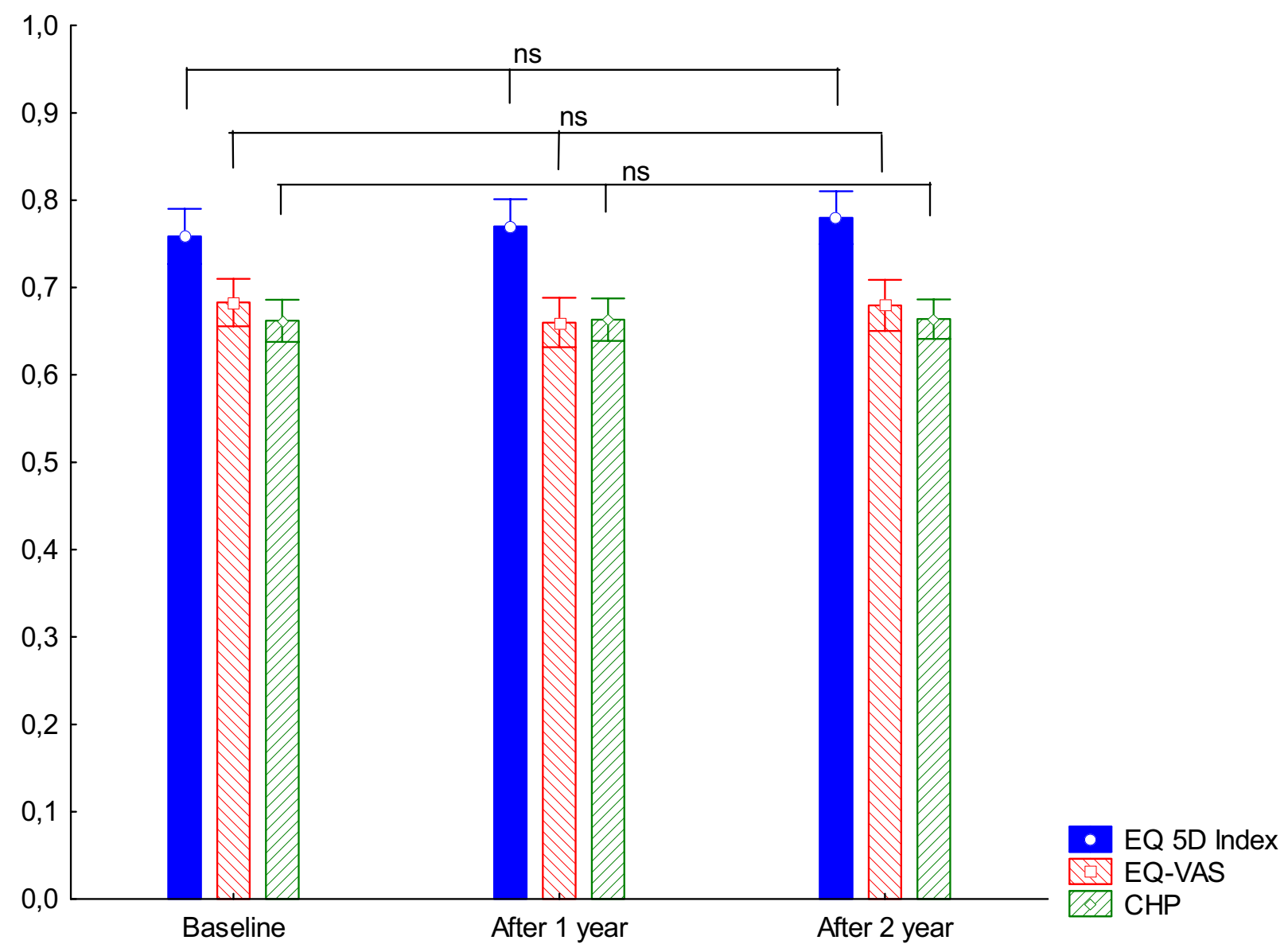

Figure 2

Health related quality of life at different times of follow up assessed by Cardiac Health Profile (CHP), EuroQol-VAS (EQ-VAS) and EuroQol-5 Dimension (EQ 5D-Index) instruments. Time trend during the two years was analysed by a repeated measurement ANOVA.

\section{Discussion}

The prevalence and severity of chest pain symptoms decreased during two years of follow-up as assessed by CCS grade in this unselected cohort of patients with CAD. However, no change was found in global HRQL as assessed by three different HRQL instruments despite the symptom reduction. This indicates that an important part of HRQL in such patients is unresponsive to change in the existence or degree of chest pain. Several explanations to this could be discussed. One possible confounder could be a change in non-cardiac morbidity. However, a separate analysis excluding patients contracting co-morbidity during the study did not change the results. The reason for the unresponsiveness could be the multidimensional construct of HRQL. As shown in table 4 the CCS grade seems to explain slightly more than one third of the variation in EQ-5D and one sixth of the variation in CHP at each time point. Looking at the main components of HRQL, CCS explained slightly less than one fifth of the variation in physical function, a minor fraction of the variation in emotional function and none of the variation in cognitive and social function at all time points. However, none of these components changed during the time period.

We have in a previous paper found that perceived cognitive function explains the major part of HRQL in this cohort [9]. However, the result of the correlation analysis showed no relation between perceived cognitive function and chest pain symptoms. Studies exploring cognitive function in CAD - either objectively measured or as perceived by the patient - are scarce. We conclude that important parts of HRQL in these patients do not depend on chest pain symptoms. This finding is strengthened by another study [23]. The effect of coronary artery bypass graft surgery was assessed by the Medical Outcome Study Short Form-36 (SF-36) questionnaire. HRQL improved in 
Table 4: Correlation between chest pain symptoms and three different global measures of health related quality of life (HRQL) respectively to four independent domains of HRQL

\begin{tabular}{|c|c|c|c|c|}
\hline$n=202$ & & Spearman Rank & $t(N-2)$ & p-value \\
\hline \multirow{3}{*}{$E Q-5 D_{\text {Index }}$} & Baseline & -0.58 & -10.02 & 0.000000 \\
\hline & After I year & -0.60 & -10.53 & 0.000000 \\
\hline & After 2 years & -0.64 & -11.82 & 0.000000 \\
\hline \multirow[t]{3}{*}{ EQ VAS $_{\text {VA }}$} & Baseline & -0.48 & -7.66 & 0.000000 \\
\hline & After I year & -0.52 & -8.64 & 0.000000 \\
\hline & After 2 years & -0.48 & -7.78 & 0.000000 \\
\hline \multirow[t]{3}{*}{ CHP } & Baseline & -0.39 & -6.06 & 0.000000 \\
\hline & After I year & -0.45 & -7.22 & 0.000000 \\
\hline & After 2 years & -0.35 & -5.33 & 0.000000 \\
\hline \multirow[t]{3}{*}{$P C_{\text {cognitive }}$} & Baseline & 0.05 & 0.70 & 0.48 \\
\hline & After I year & 0.10 & 1.39 & 0.17 \\
\hline & After 2 years & 0.09 & 1.30 & 0.20 \\
\hline \multirow{3}{*}{$P C_{\text {physical/general }}$} & Baseline & 0.41 & 6.42 & 0.000000 \\
\hline & After I year & 0.47 & 7.45 & 0.000000 \\
\hline & After 2 years & 0.43 & 6.66 & 0.000000 \\
\hline \multirow[t]{3}{*}{ PC $_{\text {social }}$} & Baseline & 0.11 & 1.63 & 0.10 \\
\hline & After I year & 0.23 & 3.32 & 0.001 \\
\hline & After 2 years & 0.08 & 1.14 & 0.25 \\
\hline \multirow{3}{*}{$P C_{\text {emotional }}$} & Baseline & 0.18 & 2.65 & 0.009 \\
\hline & After I year & 0.21 & 3.09 & 0.002 \\
\hline & After 2 years & 0.15 & 2.08 & 0.04 \\
\hline
\end{tabular}

Spearman Rank Order Correlation between existence and severity of current chest pain symptoms assessed by Canadian Cardiovascular Society classification CCS $(0-4)$ and different measures of health related quality of life

EQ-5D $D_{\text {Index }}$ denotes EuroQol 5 Dimension questionnaire transformed to an Index score (0-I)

$\mathrm{EQ}_{\text {VAS }}$ denotes EuroQol-VAS instrument (0-I)

CHP denotes the Cardiac Health Profile questionnaire (global mean score)

$\mathrm{PC}$ denotes the four independent domains of HRQL calculated from the CHP questionnaire

6 of 8 subscales. However, general health and role emotional scores did not improve in parallel to the decrease in chest pain symptoms. This finding indicates that major parts of HRQL - in patients with CAD - do not improve in parallel to an improved physical function.

A limitation of our study is that that we just assessed perceived function. However, the main aim was to evaluate time trends in illness - both in terms of HRQL and chest pain symptoms - and not primarily to address a comparison between objective function and subjective perception. Furthermore it is known that the correlation is weak between objectively measured cognitive function and perceived cognitive function [24].

Strength of our study is that HRQL was assessed both by three different global instruments and by the independent sub-domains. The response rate was also high. This should increase the robustness of our analysis and conclusions. Another strength is that this large sample of patients were followed over a good [2-year] period. Furthermore, the cohort was unselected and included both patients with and without present physical symptoms. This should increase the ability to understand the comprehensiveness of different assessment instruments and to analyse a puta- tive co variation of change in HRQL and other estimates over time.

The comprehensiveness differs between the three HRQL instruments. EQ-VAS is an individually defined global estimate. The 16 items of the CHP questionnaire taps - as compared to other frequently used instruments - a broad perspective of aspects on HRQL. It includes e g questions concerning the ability to select relevant information, and to understand, retain, express and apply knowledge in specific contexts of life. In a previous analysis we showed that questions representing cognitive function explained $43 \%$ of the HRQL variation [9]. EQ-5D includes five questions tapping five defined aspects of HRQL. It has been demonstrated that adding a cognition attribute to the standard EQ-5D system increases its comprehensiveness [25].

There was a slight but insignificant improvement of HRQL as assessed by EQ-5D $(2.6 \% ; \mathrm{p}=0.28)$. However, the power analysis showed that we could exclude a more clinically relevant improvement in HRQL by $5 \%$ or more even in the symptom driven EQ-5D. EQ-5D also showed a better global HRQL by $0.1(\mathrm{p}=0.0000)$ than CHP and EQ-VAS. An explanation might be the more narrow scope 


\section{Baseline}

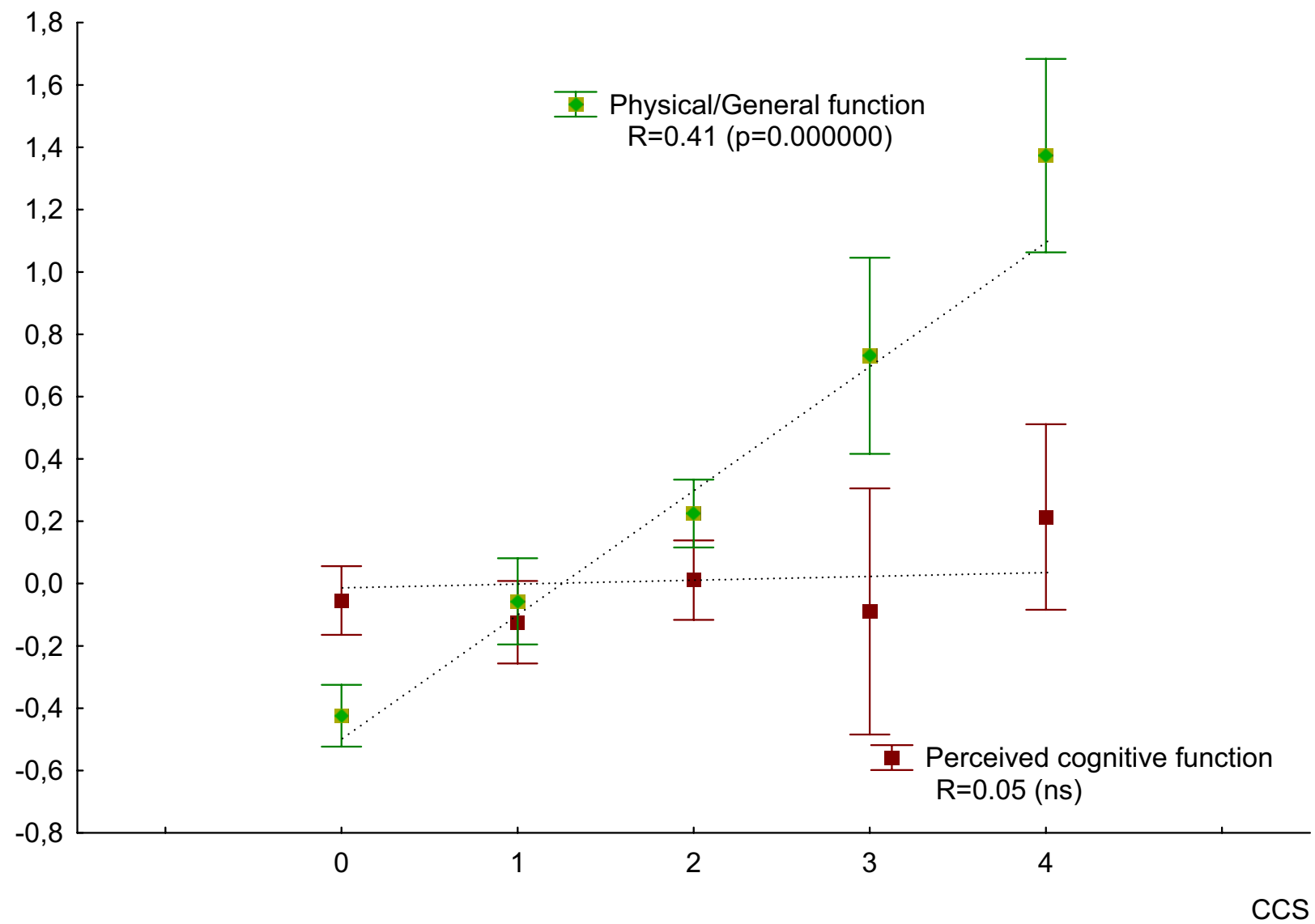

\section{Figure 3}

Covariation of chest pain grade and and the physical/general health and cognitive domains of health related quality of life at baseline. Chest pain is assessed by Canadian Cardiovascular Society classification (CCS; 0-4). Values are given as mean \pm SE. Spearman Rank order correlation analysis was used.

of EQ-5D tapping mainly physical and emotional aspects of HRQL.

It is known that physicians tend to underestimate or fail to recognise functional disabilities that are reported by their patients, especially disabilities related to social activities [26]. The physician has a disease perspective and translates the absence or decrease of chest pain symptoms to less stenotic coronary arteries with improved capacity to distribute oxygen to fulfil the demand of the working myocardial tissue. This should improve the patients' physical function. However, the patients' perception of health might have a great inertia to respond to decreased chest pain symptoms. All patients in the present study knew that their coronary arteries were affected by atherosclerosis. To many patients this knowledge might mean that they constantly are afflicted by a potentially lethal condition.
A limitation and strength of the present study is of course the unselected sample in concert with the fact that the patients are included from a single geographic area. The study addresses an important issue about responsiveness of HRQL instruments. Our findings of a low sensitivity of the HRQL instruments to change in symptom burden is interesting and challenging both from a clinical and societal perspective. The results should stimulate future research on the complex relations between objectively measured disease and subjectively perceived illness for patients with CAD.

\section{Conclusion}

The major part of assessed health related quality of life is unresponsive to a decrease in severity of chest pain symptoms in a consecutive cohort of ordinary patients with coronary artery disease. This indicates that a reduction of chest pain symptoms is not sufficient; to improve health 


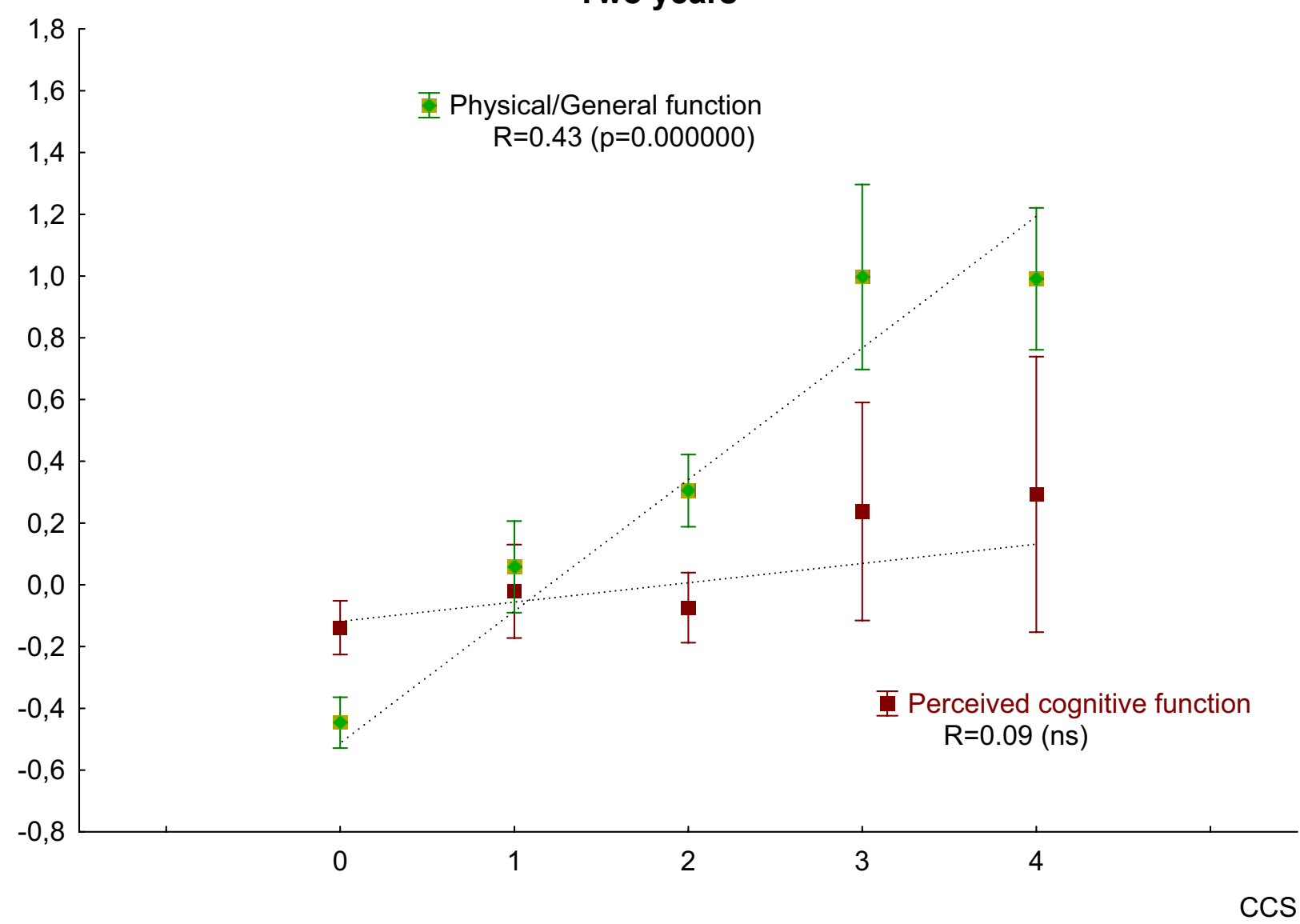

Figure 4

Covariation of chest pain grade and the physical/general health and cognitive domains of health related quality of life after two years. Chest pain is assessed by Canadian Cardiovascular Society classification (CCS; $0-4$ ). Values are given as mean \pm SE. Spearman Rank order correlation analysis was used.

related quality of life in patients with coronary artery disease. The dominating dimension of health related quality of life - cognitive function - does not differ between patients with or without chest pain symptoms. The low responsiveness of assessed health related quality of life outcome to a change in symptom burden is interesting and challenging both from a clinical and a public health perspective.

\section{Competing interests}

The author(s) declare that they have no competing interests.

\section{Authors' contributions}

$\mathrm{PH}$ and $\mathrm{AK}$ shared the conception and analysis of the study. AK was responsible for the statistical analysis, drafting and revising of the article. $\mathrm{PH}$ and $\mathrm{AK}$ shared the hypothesis and design. PH and AK are both guarantators.

\section{Acknowledgements}

We thank Katarina Patzelt-Brandt for invaluable support performing all patient interviews and data collections. This study was supported by grants from Stockholm County Council and from the Swedish Heart and Lung

Foundation.

\section{References}

I. Guidry UC, Evans JC, Larson MG, Wilson PW, Murabito JM, Levy D: Temporal trends in event rates after $Q$-wave myocardial infarction: the Framingham Heart Study. Circulation 1999, 100:2054-2059.

2. Coyne KS, Lundergan CF, Boyle D, Greenhouse SW, Draoui YC, Walker P, Ross AM: Relationship of infarct artery patency and left ventricular ejection fraction to health-related quality of life after myocardial infarction: the GUSTO-I Angiographic Study experience. Circulation 2000, 102:1245-|25I.

3. Sjoland H, Wiklund I, Caidahl K, Albertsson P, Herlitz J: Relationship between quality of life and exercise test findings after coronary artery bypass surgery. Int J Cardiol I995, 5 I:22 I-232.

4. Brorsson B, Bernstein SJ, Brook RH, Werko L: Quality of life of patients with chronic stable angina before and four years after coronary revascularisation compared with a normal population. Heart 2002, 87:|140-145. 
5. Pocock SJ, Henderson RA, Seed P, Treasure T, Hampton JR: Quality of life, employment status, and anginal symptoms after coronary angioplasty or bypass surgery. 3-year follow-up in the Randomized Intervention Treatment of Angina (RITA) Trial. Circulation 1996, 94:135-I42.

6. Brown N, Melville M, Gray D, Young T, Munro J, Skene AM, Hampton JR: Quality of life four years after acute myocardial infarction: short form 36 scores compared with a normal population. Heart 1999, 81:352-358.

7. Kiessling A, Henriksson P: Efficacy of case method learning in general practice for secondary prevention in patients with coronary artery disease: randomised controlled study. BMJ 2002, 325:877-880.

8. WHO: Proposal for the multinational monitoring of trends and determinants in cardiovascular disease (MONICA) project and protocol. Geneva, Cardiovascular Disease Unit, World Health Organisation; 1983.

9. Kiessling A, Henriksson P: Perceived cognitive function is a major determinant of health related quality of life in a nonselected population of patients with coronary artery disease - a principal components analysis. Qual Life Res 2004, |3:|62|-|63|.

10. Cox J, Naylor CD: The Canadian Cardiovascular Society grading scale for angina pectoris: is it time for refinements? Ann Intern Med 1992, i 1 7:677-683.

II. Spilker B: Quality of Life Studies: Definitions and Conceptual Issues (page 16). In Quality of life and pharmacoeconomics in clinical trials 2.th edition. Philadelphia, Lippincott-Raven; 1996:1259.

12. Wahrborg P, Emanuelsson $\mathrm{H}$ : The cardiac health profile: content, reliability and validity of a new disease-specific quality of life questionnaire. Coron Artery Dis 1996, 7:823-829.

13. Bengtsson I, Hagman M, Wedel H: Age and angina as predictors of quality of life after myocardial infarction: a prospective comparative study. Scand Cardiovasc I 200I, 35:252-258.

14. Brooks R: EuroQol: the current state of play. Health Policy 1996, 37:53-72.

15. Bjork S, Norinder A: The weighting exercise for the Swedish version of the EuroQol. Health Econ 1999, 8: I 17-126.

16. Dolan P: Modeling valuations for EuroQol health states. Med Care 1997, 35:1095-1108.

17. Macran S, Kind P: "Death" and the valuation of health-related quality of life. Med Care 200I, 39:2। 7-227.

18. Burstrom K, Johannesson M, Diderichsen F: Swedish population health-related quality of life results using the EQ-5D. Qual Life Res 200I, 10:62I-635.

19. Rabin R, de Charro F: EQ-5D: a measure of health status from the EuroQol Group. Ann Med 200I, 33:337-343.

20. Nowels D, McGloin J, Westfall JM, Holcomb S: Validation of the EQ-5D quality of life instrument in patients after myocardial infarction. Qual Life Res 2005, 14:95-105.

21. Armitage P, Berry G: Statistical methods in medical research. 3rd edition. Oxford ; Boston, Blackwell Scientific Publications; 1994:xi, 620.

22. Glantz SA, Slinker BK: Primer of applied regression and analysis of variance. New York, McGraw-Hill Health Professions Division; 1990:xxii, 777.

23. Kiebzak GM, Pierson LM, Campbell M, Cook JW: Use of the SF36 general health status survey to document health-related quality of life in patients with coronary artery disease: effect of disease and response to coronary artery bypass graft surgery. Heart Lung 2002, 3 I:207-2I3.

24. Dijkstra JB, Jolles J: Postoperative cognitive dysfunction versus complaints: a discrepancy in long-term findings. Neuropsychol $\operatorname{Rev} 2002$, I 2: I- 14

25. Krabbe PF, Stouthard ME, Essink-Bot ML, Bonsel GJ: The effect of adding a cognitive dimension to the EuroQol multiattribute health-status classification system. J Clin Epidemiol 1999 , 52:293-30I.

26. Calkins DR, Rubenstein LV, Cleary PD, Davies AR, Jette AM, Fink A, Kosecoff J, Young RT, Brook RH, Delbanco TL: Failure of physicians to recognize functional disability in ambulatory patients. Ann Intern Med 199I, I I 4:45I-454.
Publish with Biomed Central and every scientist can read your work free of charge

"BioMed Central will be the most significant development for disseminating the results of biomedical research in our lifetime. "

Sir Paul Nurse, Cancer Research UK

Your research papers will be:

- available free of charge to the entire biomedical community

- peer reviewed and published immediately upon acceptance

- cited in PubMed and archived on PubMed Central

- yours - you keep the copyright

Submit your manuscript here:

http://www.biomedcentral.com/info/publishing_adv.asp
BiolMedcentral 